\title{
Approximation of the Likelihood Ratio Statistics in Competing Risks Model Under Informative Random Censorship From Both Sides
}

\author{
Abdurahim A. Abdushukurov \\ National University of Uzbekistan \\ Department of Theory Probability and Mathematical Statistics \\ 100178, Tashkent, Uzbekistan \\ a_abdushukurov@rambler.ru
}

Nargiza S. Nurmuhamedova

National University of Uzbekistan

Department of Theory Probability and Mathematical Statistics

100178, Tashkent, Uzbekistan

rasulova_nargiza@mail.ru

\begin{abstract}
It is clear that the likelihood ratio statistics plays an important role in theories of asymptotical estimation and hypothesis testing. The aim of the paper is to investigate the asymptotic properties of likelihood ratio statistics in competing risks model with informative random censorship from both sides. We prove the approximation version of the locally asymptotically normality of the likelihood ratio statistics. The results have asymptotic representation of the likelihood ratio statistics using the strong approximation method where local asymptotic normality is obtained as a consequence.
\end{abstract}

Keywords: Likelihood ratio statistics, Competing risks model, Locally asymptotically normality, Random censoring.

\section{Introduction}

The most important property of statistical models is the locally asymptotically normality (LAN) of likelihood ratio statistics (LRS) of a regular statistical experiment. The essence of the LAN is that the LRS of model can be approximated by functions of the from $\exp \left\{u \omega_{n, \theta}-\frac{1}{2} u^{2}\right\}$, where $\omega_{n, \theta}$ - asymptotically (at $n \rightarrow \infty$ ) normal with the parameters $(0,1)$ random variables (r.v.). LAN in the case of independent and identically distributed (i.i.d.) observations studied by A.Wald, L.Le Kam and J. Hajek (for details see [6-11]). In [3-5] established results of strong approximation for LRS by stochastic integrals in competing risks model (CRM) for random censoring of observations on the right and from both sides. In this paper we consider similar problems in the CRM, when random censoring from both sides is informative.

\section{Informative model and its characterization}

Following [3-5], we consider the CRM. Let $X$ - r.v. with values in a measurable space $(\mathrm{X}, \mathrm{B})$, where $\mathrm{X} \subseteq R^{1}, \mathrm{~B}=\sigma(\mathrm{X})$. Consider the disjoint events $\left\{A^{(1)}, \ldots, A^{(k)}\right\}$ (or at least 
$\left.P\left(A^{(i)} \cap A^{(j)}\right)=0, \quad i \neq j, \quad i, j=\overline{1, l}\right)$, such that $P\left(\bigcup_{i=1}^{k} A^{(i)}\right)=1$. In CRM our interest is concentrated on joint properties of r.v. $X$ and events $\left\{A^{(i)}, i=\overline{1, k}\right\}$. Let $\left\{\delta^{(i)}=I\left(A^{(i)}\right), i=\overline{1, k}\right\}$ - indicators of these events, and joint distribution of the vector $\left(X, \delta^{(1)}, \ldots, \delta^{(k)}\right)$ depends on unknown parameter $\theta \in \Theta$ :

$$
Q_{\theta}\left(x, y^{(1)}, \ldots, y^{(k)}\right)=P_{\theta}\left(X<x, \delta^{(1)}=y^{(1)}, \ldots, \delta^{(k)}=y^{(k)}\right),
$$

where $x \in R^{1}, y^{(i)} \in\{0,1\}, i=\overline{1, k}$ and $\Theta$ an open set in $R^{1}$ for simplicity $\theta$ is scalar parameter.

Let $H(x ; \theta)=P_{\theta}(X \leq x)$ and $H^{(i)}(x ; \theta)=P_{\theta}\left(X<x, \delta^{(i)}=1\right)$ - marginal distributions of r.v. $X \quad$ and $\left(X, \delta^{(i)}\right), i=\overline{1, k} \quad$ respectively. Since $\quad \delta^{(1)}+\ldots+\delta^{(k)}=1$, then $H^{(1)}(x ; \theta)+\ldots+H^{(k)}(x ; \theta)=H(x ; \theta)$ - for all $(x ; \theta) \in R^{1} \times \Theta$. Let subdistributions $H^{(i)}$ are absolutely continuous and have densities $h^{(i)}$. Then there is a density $h$ of d.f. $H$ and for all $(x ; \theta) \in R^{1} \times \Theta: h(x ; \theta)=h^{(1)}(x ; \theta)+\ldots+h^{(k)}(x ; \theta)$. Assume that the r.v. $X$ subject to random censoring from both sides by pair of r.v.-s $(L, Y)$ with absolutely continuous d.f.-s $K(x ; \theta)=P_{\theta}(L<x)$ and $G(y ; \theta)=P_{\theta}(Y<y)$. It also assumes that the r.v.-s $\{X, L, Y\}-$ are independent, and censoring is informative, i.e., d.f.-s $K$ and $G$ expressed in terms of d.f. $H$ by the following formulas for all $(x ; \theta) \in R^{1} \times \Theta$ :

$$
\left\{\begin{array}{l}
1-G(x ; \theta)=(1-H(x ; \theta))^{\alpha} \\
K(x ; \theta)=\left[1-(1-H(x ; \theta))^{\alpha+1}\right]^{\beta}
\end{array}\right.
$$

where $\alpha$ and $\beta$ are positive and unknown nuisance parameters independent of $\theta$.

Let $k$ and $g$ are density functions corresponding to the d.f. $K$ and $G$ respectively. Then according to (1)

$$
\begin{aligned}
& k(x ; \theta)=\beta\left[1-(1-H(x ; \theta))^{\alpha+1}\right]^{\beta-1} \cdot(\alpha+1)(1-H(x ; \theta))^{\alpha-1} h(x ; \theta), \\
& g(x ; \theta)=\alpha(1-H(x ; \theta))^{\alpha-1} h(x ; \theta) .
\end{aligned}
$$

Let $\tau_{H}=\inf \{x: H(x ; \theta)>0\}$ and $T_{H}=\sup \{x: H(x ; \theta)<1\}$. Then from $(1), \tau_{H}=\tau_{K}=\tau_{L}, T_{H}=T_{K}=T_{L}$, and a densities (2) are positive on the set $\left[\tau_{H} ; T_{H}\right]$.

Let $\left\{\left(X_{j}, L_{j}, Y_{j}, A_{j}^{(1)}, \ldots, A_{j}^{(k)}\right), j \geq 1\right\}$ - a sequence of independent replicas of the aggregate $\left(X, L, Y, A^{(1)}, \ldots, A^{(k)}\right)$. On $n$ - th stage of the experiment we observe the sample of size $n$ : $\bar{\square}^{(n)}=\left(\bar{Z}_{1}, \ldots, \bar{Z}_{n}\right), \quad$ where $\quad \bar{Z}_{j}=\left(Z_{j} ; \Delta_{j}^{(-1)}, \Delta_{j}^{(0)}, \Delta_{j}^{(1)}, \ldots, \Delta_{j}^{(k)}\right), \quad Z_{j}=L_{j} \vee\left(X_{j} \wedge Y_{j}\right)=\max \left(L_{j}, \min \left(X_{j}, Y_{j}\right)\right)$, $\Delta_{j}^{(l)}=I\left(D_{j}^{(l)}\right), \quad l=-1,0,1, \ldots, k \quad$ and $\quad$ events $\quad D_{j}^{(-1)}=\left\{\omega: X_{j}(\omega) \wedge Y_{j}(\omega)<L_{j}(\omega)\right\}$, $D_{j}^{(0)}=\left\{\omega: L_{j}(\omega) \leq Y_{j}(\omega)<X_{j}(\omega)\right\}, \quad D_{j}^{(i)}=A_{j}^{(i)} \cap \quad \cap\left\{\omega: L_{j}(\omega) \leq X_{j}(\omega) \leq Y_{j}(\omega)\right\}, \quad i=1, \ldots k . \quad \mathrm{We}$ 
introduce d.f.-s $M(x ; \theta)=P_{\theta}\left(X_{j} \wedge Y_{j} \leq x\right)=1-(1-G(x ; \theta)) \cdot(1-H(x ; \theta))$ and $N(x ; \theta)=P_{\theta}\left(Z_{j} \leq x\right)=$ $K(x ; \theta) M(x ; \theta)$, as well as subdistributions $T^{(l)}(x ; \theta)=P_{\theta}\left(Z_{j} \leq x ; \Delta_{j}^{(l)}=1\right), l=-1,0,1, \ldots, k$. It is easy to see that

$$
\begin{aligned}
& T^{(-1)}(x ; \theta)=\int_{-\infty}^{x} M(u ; \theta) d K(u ; \theta), \\
& T^{(0)}(x ; \theta)=\int_{-\infty}^{x} K(u ; \theta)(1-H(u ; \theta)) d G(u ; \theta), \\
& T^{(i)}(x ; \theta)=\int_{-\infty}^{x} K(u ; \theta)(1-G(u ; \theta)) d H^{(i)}(u ; \theta), i=\overline{1, k} .
\end{aligned}
$$

Then

$$
\sum_{i=1}^{k} T^{(i)}(x ; \theta)=\int_{-\infty}^{x} K(u ; \theta)(1-G(u ; \theta)) d H(u ; \theta)=T(x ; \theta)
$$

and

$T^{(-1)}(x ; \theta)+T^{(0)}(x ; \theta)+T^{(1)}(x ; \theta)+\ldots++T^{(k)}(x ; \theta)=N(x ; \theta)$. Let $\gamma=\frac{1}{1+\alpha}$ and $\lambda=\frac{1}{1+\beta}$. Then according to (1), by direct calculation of subdistributions (3), we have

$$
\begin{gathered}
T^{(-1)}(x ; \theta)=\int_{-\infty}^{x} M(u ; \theta) d(M(u ; \theta))^{\beta}=\frac{\beta}{1+\beta}(M(x ; \theta))^{\beta+1}=(1-\lambda) N(x ; \theta) \\
T^{(0)}(x ; \theta)=-\int_{-\infty}^{x}(M(u ; \theta))^{\beta}(1-H(u ; \theta)) d(1-H(u ; \theta))^{\alpha}=-\frac{\alpha}{1+\alpha} \int_{-\infty}^{x}(M(u ; \theta))^{\beta} d(1-H(u ; \theta))^{\alpha+1}= \\
=-\frac{\alpha}{1+\alpha} \int_{-\infty}^{x}(M(u ; \theta))^{\beta} d M(u ; \theta)=(1-\lambda) \gamma N(x ; \theta) \\
T(x ; \theta)=-\frac{1}{1+\alpha} \int_{-\infty}^{x}(M(u ; \theta))^{\beta} d(1-H(u ; \theta))^{\alpha+1}=-\frac{1}{1+\alpha} \int_{-\infty}^{x}(M(u ; \theta))^{\beta} d M(u ; \theta)=\lambda \gamma N(x ; \theta)
\end{gathered}
$$

Informative model under simple random censoring from both sides without competing risks was first introduced in [1,2]. Following [1,2], we prove a theorem characterizing the model (1) by independence of r.v.-s $Z_{j}$ and vectors $\left(\Delta_{j}^{(-1)}, \Delta_{j}^{(0)}, \Delta_{j}\right)$, where $\Delta_{j}=\Delta_{j}^{(1)}+\ldots+\Delta_{j}^{(k)}$.

Theorem 2.1. Model (1) holds if and only if the r.v. $Z_{j}$ and vector $\left(\Delta_{j}^{(-1)}, \Delta_{j}^{(0)}, \Delta_{j}\right)$ are independent for each $j \geq 1$.

Proof of the Theorem 2.1. Suppose that the formulas (1) are hold. Then we have the representation (4) - (6) for subdistributions. Tending to the limit under $x \rightarrow \infty$ in these formulas, in particular for all $\theta \in \Theta$ we have

$$
P_{\theta}\left(\Delta_{j}^{(-1)}=1\right)=1-\lambda, P_{\theta}\left(\Delta_{j}^{(0)}=1\right)=(1-\gamma) \lambda, P_{\theta}\left(\Delta_{j}=1\right)=\lambda \gamma .
$$


Introduce the events $A=\left\{Z_{j} \leq x\right\}$ and $\left\{B_{-1}, B_{0}, B_{1}\right\}$, where $B_{m}=\left\{\Delta_{j}^{(m)}=1\right\}, m=-1,0$ and $B_{1}=\left\{\Delta_{j}=1\right\}$. Then from (4)-(7) it follows the independence of events $A$ and $\left\{B_{m}, m=-1,0,1\right\}$. Similarly, we can show independence of other combinations of these events and their rejections. This shows that the r.v. $Z_{j}$ and vector $\left(\Delta_{j}^{(-1)}, \Delta_{j}^{(0)}, \Delta_{j}\right)$ are independent. Conversely, let r.v. $Z_{j}$ and vector $\left(\Delta_{j}^{(-1)}, \Delta_{j}^{(0)}, \Delta_{j}\right)$ are independent. In particular, from independence of $Z_{j}$ and $\Delta_{j}^{(-1)}$ for all $x>\tau_{K}$ we have

$$
\begin{aligned}
& K(x ; \theta)=\exp \left\{-\int_{x}^{+\infty} \frac{d T^{(-1)}(u ; \theta)}{M(u ; \theta) K(u ; \theta)}\right\}=\exp \left\{-P_{\theta}\left(\Delta_{j}^{(-1)}=1\right) \int_{x}^{+\infty} \frac{d N(u ; \theta)}{N(u ; \theta)}\right\}= \\
& {[M(x ; \theta) K(x ; \theta)]^{P_{\theta}\left(\Delta_{j}^{(-1)}=1\right)} .}
\end{aligned}
$$

From here the second representation in (1) follows under $\beta=P_{\theta}\left(\Delta_{j}^{(-1)}=1\right)\left(P_{\theta}\left(\Delta_{j}^{(-1)} \neq 1\right)\right)$. On the other hand, since independence of $Z_{j}$ and $\Delta_{j}^{(0)}$ for all $x>\tau_{G}$,

$$
1-G(x ; \theta)=\exp \left\{-\int_{-\infty}^{x} \frac{d T^{(0)}(u ; \theta)}{K(u ; \theta)(1-H(u ; \theta))}\right\}=\exp \left\{-P_{\theta}\left(\Delta_{j}^{(0)}=1\right) \int_{-\infty}^{x} \frac{d N(u ; \theta)}{K(u ; \theta)-N(u ; \theta)}\right\},
$$

also from independence of $Z_{j}$ and $\Delta_{j}$ for all $x>\tau_{H}$ we have

$$
1-H(x ; \theta)=\exp \left\{-\int_{-\infty}^{x} \frac{d T(u ; \theta)}{K(u ; \theta)-N(u ; \theta)}\right\}=\exp \left\{-P_{\theta}\left(\Delta_{j}=1\right) \int_{-\infty}^{x} \frac{d N(u ; \theta)}{K(u ; \theta)-N(u ; \theta)}\right\} .
$$

Now from (8) and (9) for all $(x ; \theta) \in R^{1} \times \Theta$

$$
-\log (1-G(x ; \theta))=-\alpha \log (1-H(x ; \theta)),
$$

where $\alpha=P_{\theta}\left(\Delta_{j}^{(0)}=1\right)\left(P_{\theta}\left(\Delta_{j}=1\right)\right)^{-1}$, which shows the validity of first formula in (1). Theorem 2.1 is proved.

Let $p^{(-1)}=P_{\theta}\left(\Delta_{j}^{(-1)}=1\right)$ and $p=P_{\theta}\left(\Delta_{j}=1\right)$. Then $\gamma=\frac{1}{1+\alpha}=\frac{p}{1-p^{(-1)}}, \lambda=\frac{1}{1+\beta}=1-p^{(-1)}$ and therefore these parameters are estimated by the statistics

$$
\gamma_{n}=\frac{p_{n}}{1-p_{n}^{(-1)}} \text { and } \lambda_{n}=1-p_{n}^{(-1)}
$$

where $p_{n}^{(-1)}=\frac{1}{n} \sum_{j=1}^{n} \Delta_{j}^{(-1)}, p_{n}=\frac{1}{n} \sum_{j=1}^{n} \Delta_{j}=\frac{1}{n} \sum_{j=1}^{n} \sum_{i=1}^{k} \Delta_{j}^{(i)}$.

\section{Approximation of the LRS}


Let $\left(\mathrm{Y}^{(n)}, \mathrm{U}^{(n)}, Q_{\theta}^{(n)}\right)$ - denote the sequence of statistical experiments generated by observations $\bar{\square}^{(n)}$, where $\mathrm{Y}^{(n)}=\left\{\mathrm{X} \times\{0,1\}^{(k+2)}\right\}^{(n)}, \mathrm{U}^{(n)}=\sigma\left(\mathrm{Y}^{(n)}\right), Q_{\theta}^{(n)}$ - distribution on $\left(\mathrm{Y}^{(n)}, \mathrm{U}^{(n)}\right)$. The family of measures $\left\{Q_{\theta}^{(n)}, \theta \in \Theta, n \geq 1\right\}$ is absolutely continuous with respect to measure $v^{(n)}=v_{1} \times \ldots \times v_{n}$, where $d v_{m}=d x_{m} \times \varepsilon_{y_{m}^{(-1)}} \times \ldots \times \varepsilon_{y_{m}^{(k)}}, \quad \varepsilon_{y_{m}^{(l)}}-$ counting measures concentrated at a point $y_{m}^{(l)} \in\{0,1\}, l=-1,0,1, \ldots, k ; m=\overline{1, n}$ and its density is given by

$$
\begin{gathered}
p_{n}\left(\bar{\square}^{(n)} ; \theta\right)=\frac{d Q_{\theta}^{(n)}\left(\bar{\square}^{(n)}\right)}{d v^{(n)}\left(\bar{\square}^{(n)}\right)}=\prod_{j=1}^{n}\left\{\left[M\left(Z_{j} ; \theta\right) k\left(Z_{j} ; \theta\right)\right]^{\Delta_{j}^{(-1)}} \cdot\left[K\left(Z_{j} ; \theta\right) \cdot\left(1-H\left(Z_{j} ; \theta\right)\right) g\left(Z_{j} ; \theta\right)\right]^{\Delta_{j}^{(0)}} .\right. \\
\left.\cdot \prod_{i=1}^{k}\left[K\left(Z_{j} ; \theta\right)\left(1-G\left(Z_{j} ; \theta\right)\right) h^{(i)}\left(Z_{j} ; \theta\right)\right]^{\Delta_{j}^{(i)}}\right\} .
\end{gathered}
$$

Now, by using (1) and (2) after simple algebra the density (12) can be represented as follows:

$$
p_{n}\left(\bar{\square}^{(n)} ; \theta\right)=\chi_{n}(\alpha ; \beta) \prod_{j=1}^{n}\left\{\left[t\left(Z_{j} ; \theta\right)\right]^{1-\Delta_{j}} \cdot \prod_{i=1}^{k}\left[t^{(i)}\left(Z_{j} ; \theta\right)\right]^{\Delta_{j}^{(i)}}\right\},
$$

where $\chi_{n}(\alpha ; \beta)=[\beta(\alpha+1)]_{j=1}^{n} \Delta_{j}^{(-1)} \cdot \alpha^{\sum_{j=1}^{n} \Delta_{j}^{(0)}}$ - independent of $\theta, t(x ; \theta)=\sum_{i=1}^{n} t^{(i)}(x ; \theta)$ - density of subdistribution $T(x ; \theta)$ and $t^{(i)}(x ; \theta)==h^{(i)}(x ; \theta) K(x ; \theta)(1-G(x ; \theta))=\frac{\partial T^{(i)}(x ; \theta)}{\partial x}, \quad i=\overline{1, k}$. Note that according to the representation (13) statistics $\left(Z_{1}, \ldots, Z_{n} ; \Delta_{1}, \ldots, \Delta_{n}\right)$ is a sufficient statistic for this informative model. From (13) one can expect that the LAN property for this model depends on the properties of the density $t^{(i)}, i=1, \ldots, k$. Because, $t^{(i)}(x ; \theta)=h^{(i)}(x ; \theta) \cdot \cdot[1-H(x ; \theta)]^{\alpha}[1-(1-H(x ; \theta))]^{\beta}$, then have $t^{(i)}(x ; \theta) \leq h^{(i)}(x ; \theta)$ and $t(x ; \theta) \leq h(x ; \theta)$ for all $i=\overline{1, k},(x ; \theta) \in R^{1} \times \Theta$. We need some regularity conditions:

(C1) Supports $N_{h^{(i)}}=\left\{x: h^{(i)}(x ; \theta)>0\right\}, \quad i=\overline{1, k}$ are independent on parameter $\theta$ and $\bigcap_{i=1}^{k} N_{h^{(i)}} \neq \varnothing$

(C2) For any $\theta_{1}, \theta_{2} \in \Theta, \theta_{1} \neq \theta_{2}$ and $x \in N_{h^{(i)}}: h^{(i)}\left(x ; \theta_{1}\right) \neq h^{(i)}\left(x ; \theta_{2}\right), i=1, \ldots, k$;

(C3) There are finite for all $x$ derivatives $\partial^{l} h^{(i)}(x ; \theta) / \partial \theta^{l}, \quad l=1,2 ; i=\overline{1, k}$ and

$$
\int_{-\infty}^{\infty}\left|\partial^{l} h^{(i)}(x ; \theta) / \partial \theta^{l}\right| d x<\infty, l=1,2 ; i=1, \ldots, k
$$

(C4) Functions $\left\{\frac{\partial \log t^{(i)}(x ; \theta)}{\partial \theta}, i=\overline{1, k}, \frac{\partial \log t(x ; \theta)}{\partial \theta}\right\}$ are of bounded variations;

(C5) Fisher information 


$$
J(\theta)=\sum_{i=1}^{k} \int_{-\infty}^{\infty}\left(\frac{\partial \log t^{(i)}(x ; \theta)}{\partial \theta}\right)^{2} d T^{(i)}(x ; \theta)+\int_{-\infty}^{\infty}\left(\frac{\partial \log t(x ; \theta)}{\partial \theta}\right)^{2} d\left[T^{(-1)}(x ; \theta)+T^{(0)}(x ; \theta)\right]
$$

is finite and positive at the point $\theta=\theta_{0}$.

We define the empirical estimates of the distributions $N(x ; \theta), T^{(m)}(x ; \theta), m=-1,0,1, \ldots, k$ and $T(x ; \theta)$ :

$$
\begin{aligned}
& N_{n}(x)=\frac{1}{n} \sum_{j=1}^{n} I\left(Z_{j}<x\right)=T_{n}^{(-1)}(x)+T_{n}^{(0)}(x)+T_{n}(x), T_{n}^{(m)}(x)=\frac{1}{n} \sum_{j=1}^{n} \Delta_{j}^{(m)} I\left(Z_{j}<x\right), m=-1,0,1, \ldots, k, \\
& T_{n}(x)=\frac{1}{n} \sum_{i=1}^{k} T_{n}^{(i)}(x) .
\end{aligned}
$$

We denote $N\left(x ; \theta_{0}\right)=N(x), T^{(m)}\left(x ; \theta_{0}\right)=T^{(m)}(x), \quad m=-1,0,1, \ldots, k$ and $T\left(x ; \theta_{0}\right)=T(x)$, where $\theta_{0} \in \Theta$ is true value of $\theta$. Let $\theta_{n}=\theta_{0}+\frac{u}{\sqrt{n}} \in \Theta$, where $u \in R^{1}$. We introduce the LRS

$$
L_{n}(u)=\frac{p_{n}\left(\tilde{Z}^{(n)} ; \theta_{n}\right)}{p_{n}\left(\tilde{Z}^{(n)} ; \theta_{0}\right)}=\prod_{j=1}^{n}\left\{\prod_{i=1}^{k}\left[\frac{t^{(i)}\left(Z_{j} ; \theta_{n}\right)}{t^{(i)}\left(Z_{j} ; \theta_{0}\right)}\right]^{\Delta_{j}^{(i)}} \cdot\left[\frac{t\left(Z_{j} ; \theta_{n}\right)}{t\left(Z_{j} ; \theta_{0}\right)}\right]^{1-\Delta_{j}}\right\},
$$

and its logarithm

$$
\begin{aligned}
\log L_{n}(u) & =\sum_{j=1}^{n}\left\{\sum_{i=1}^{k} \Delta_{j}^{(i)} \log \left[\frac{t^{(i)}\left(Z_{j} ; \theta_{n}\right)}{t^{(i)}\left(Z_{j} ; \theta_{0}\right)}\right]+\left(1-\Delta_{j}\right) \log \left[\frac{t\left(Z_{j} ; \theta_{n}\right)}{t\left(Z_{j} ; \theta_{0}\right)}\right]\right\}= \\
& =n\left\{\sum_{i=1}^{k} \int_{-\infty}^{+\infty} \log \left[\frac{t^{(i)}\left(x ; \theta_{n}\right)}{t^{(i)}\left(x ; \theta_{0}\right)}\right] d T_{n}^{(i)}(x)\right\}+\int_{-\infty}^{+\infty} \log \left[\frac{t\left(x ; \theta_{n}\right)}{t\left(x ; \theta_{0}\right)}\right] d\left[T_{n}^{(-1)}(x)+T_{n}^{(0)}(x)\right] .
\end{aligned}
$$

We have

Theorem 3.1. Let the regularity conditions (C1) - (C5) are hold. Then for each $u \in R^{1}$ for the LRS we have representation

$$
L_{n}(u)=\exp \left\{u W_{n}-\frac{u^{2}}{2} J\left(\theta_{0}\right)+R_{n}(u)\right\}
$$

where

$$
\begin{aligned}
& W_{n}=\sum_{i=1}^{k} \int_{-\infty}^{\infty} \frac{\partial \log t^{(i)}\left(x ; \theta_{0}\right)}{\partial \theta} d n^{-1 / 2} \tilde{W}_{i}\left(T^{(i)}(x) ; n\right)+ \\
& \int_{-\infty}^{\infty} \frac{\partial \log t\left(x ; \theta_{0}\right)}{\partial \theta} d n^{-1 / 2}\left[\tilde{W}_{-1}\left(T^{(-1)}(x) ; n\right)+\tilde{W}_{0}\left(T^{(0)}(x) ; n\right)\right],
\end{aligned}
$$

and $R_{n}(u) \rightarrow 0$ at $n \rightarrow \infty$ in $Q_{\theta_{0}}^{(n)}$-probability. Here $\left\{\tilde{W}_{m}(y ; n), m=-1,0,1, \ldots, k\right\}$ are twoparametrical Wiener processes on $[0,1] \times(0, \infty)$, and the components of the vector $\left(\tilde{W}_{-1}, \tilde{W}_{0}, \tilde{W}_{1}, \ldots, \tilde{W}_{k}\right)$ are independent. 
Remark 3.1. In view of the properties of processes $\left\{\tilde{W}_{m}, m=-1,0,1, \ldots, k\right\}$ the r.v. $W_{n}$ is the sum of independent stochastic integrals of Ito, and for each $n \geq 1$ :

$$
\mathscr{L}\left(W_{n} / Q_{\theta_{0}}^{(n)}\right) \stackrel{D}{=} N\left(0, J\left(\theta_{0}\right)\right) .
$$

Hence, the theorem 3.1 one can written as follows: for each $u \in R^{1}$

$$
L_{n}(u)=u J^{1 / 2}\left(\theta_{0}\right) \zeta-\frac{u^{2}}{2} J\left(\theta_{0}\right)+R_{n}(u),
$$

where $\zeta^{D}=N(0,1)$.

To prove Theorem 3.1 we need in some auxiliary results. Define the empirical processes of $x \in R^{1}$ :

$$
\alpha_{n}(x)=\sqrt{n}\left(N_{n}(x)-N(x)\right), \quad \tilde{\alpha}_{i n}(x)=\sqrt{n}\left(T_{n}^{(i)}(x)-T^{(i)}(x)\right), i=-1,0,1, \ldots, k .
$$

We formulate a theorem which is an generalized analogue of Komlos, Major and Tusnady's type result. Let $\|v\|^{k+3}=\max \left\{\left|v_{-2}\right|,\left|v_{-1}\right|,\left|v_{0}\right|,\left|v_{1}\right|, \ldots,\left|v_{k}\right|\right\}$ - maximum-norm of a vector $v=\left(v_{-2}, v_{-1}, v_{0}, v_{1}, \ldots, v_{k}\right) \in R^{k+3}$. From [2, §1.9] we give the following statement of Burke, Csörgö and Horvath.

Theorem A [2]. On the probability space $(\Omega, \mathcal{A}, \mathcal{P})$ one can construct $k+3$ twoparametrical processes $K(x ; n), \tilde{K}_{-1}(x ; n), \tilde{K}_{0}(x ; n), \tilde{K}_{1}(x ; n), \ldots, \tilde{K}_{k}(x ; n)$ such that for the vector $\alpha_{n}(v)=\left(\alpha_{n}\left(v_{-2}\right), \quad \tilde{\alpha}_{-1 n}\left(v_{-1}\right), \tilde{\alpha}_{0 n}\left(v_{0}\right), \tilde{\alpha}_{1 n}\left(v_{1}\right), \ldots, \tilde{\alpha}_{k n}\left(v_{k}\right)\right), v=\left(v_{-2}, v_{-1}, v_{0}, \quad v_{1}, \ldots, v_{k}\right) \in R^{k+3}$, we have approximation

$$
\sup _{v \in R^{k+3}}\left\|\alpha_{n}(v)-n^{-1 / 2} \Gamma(v ; n)\right\|^{k+3} \stackrel{\text { a.s. }}{=} O\left(n^{-1 / 2}(\log n)^{2}\right),
$$

where $\Gamma(v ; n)=\left(K\left(v_{-2} ; n\right), \tilde{K}_{-1}\left(v_{-1} ; n\right), \tilde{K}_{0}\left(v_{0} ; n\right), \tilde{K}_{1}\left(v_{1} ; n\right), \ldots, \quad \tilde{K}_{k}\left(v_{k} ; n\right)\right)$ is $k+3$ - dimensional Gaussian process having the same covariance structure as a vector $n^{-1 / 2} \alpha_{n}(v)$, i.e. $E \Gamma(v ; n)=0$ and for any $i, j=-1, \overline{0, k}, i \neq j$ :

$$
\begin{aligned}
& E K(x ; n) K(y ; m)=(n \wedge m)\{N(x) \wedge N(y)-N(x) N(y)\}, \\
& E \tilde{K}_{i}(x ; n) \tilde{K}_{i}(y ; m)=(n \wedge m)\left\{T^{(i)}(x) \wedge T^{(i)}(y)-T^{(i)}(x) T^{(i)}(y)\right\}, \\
& E \tilde{K}_{i}(x ; n) \tilde{K}_{j}(y ; m)=(n \wedge m)\left\{-T^{(i)}(x) T^{(j)}(y)\right\}, \\
& E \tilde{K}_{i}(x ; n) K(y ; m)==(n \wedge m)\left\{T^{(i)}(x) \wedge T^{(i)}(y)-T^{(i)}(x) N(y)\right\} .
\end{aligned}
$$

Lemma 3.1. Let the regularity condition $(C 1)-(C 3)$ are hold. Then there exist finite derivatives $\partial^{l} t^{(i)}(x ; \theta) / \partial \theta^{l}, l=1,2 ; i=\overline{1, k}$ for all $(x ; \theta) \in R^{1} \times \Theta$

$$
\int_{-\infty}^{\infty}\left|\frac{\partial^{l} t^{(i)}(x ; \theta)}{\partial \theta^{l}}\right| d x<\infty, l=1,2 ; i=1, \ldots, k ;
$$

and 


$$
\sum_{i=1}^{k} E_{\theta}\left[\Delta_{1}^{(i)} \frac{\partial \log t^{(i)}\left(Z_{1} ; \theta\right)}{\partial \theta}\right]+E_{\theta}\left[\left(1-\Delta_{1}\right) \frac{\partial \log t\left(Z_{1} ; \theta\right)}{\partial \theta}\right]=0 .
$$

Proof of the Lemma 3.1. With regard to the expression of subdensity $t^{(i)}$ through $h^{(i)}, i=\overline{1, k}$, it is easy to see that the regularity condition $(\mathrm{C} 1)-(\mathrm{C} 3)$ are hold for them, and in particular (18) also holds. Moreover, taking into account the independence of r.v. $Z_{1}$ and $\left(\Delta_{1}^{(-1)}, \Delta_{1}^{(0)}, \Delta_{1}\right)$ (Theorem 2.1) we have

$$
\begin{aligned}
& E_{\theta}\left[\frac{1}{n} \frac{\partial \log p_{n}\left(\tilde{Z}^{(n)} ; \theta\right)}{\partial \theta}\right]=\sum_{i=1}^{k} \int_{-\infty}^{+\infty} \frac{\partial \log t^{(i)}(x ; \theta)}{\partial \theta} d T^{(i)}(x ; \theta)+ \\
& +(1-p) \int_{-\infty}^{+\infty} \frac{\partial \log t(x ; \theta)}{\partial \theta} d T(x ; \theta)=\frac{\partial}{\partial \theta} \sum_{i=1}^{k} \int_{-\infty}^{+\infty} t^{(i)}(x ; \theta) d x+ \\
& +(1-p) \frac{\partial}{\partial \theta} \int_{-\infty}^{+\infty} t(x ; \theta) d x=(2-p) \frac{\partial}{\partial \theta} T(\infty ; \theta)=(2-p) \frac{\partial}{\partial \theta}(\lambda \gamma)=0,
\end{aligned}
$$

as $\gamma$ and $\lambda$ assumed to be independent of $\theta$. Lemma 3.1 is proved.

Lemma 3.2. Under the conditions of Theorem 3.1 when $n \rightarrow \infty$ for each $u \in R^{1}$ we have

$$
\begin{aligned}
& L_{n}(u)=u \sum_{i=1}^{k} \sqrt{n} \int_{-\infty}^{\infty} \frac{\partial \log t^{(i)}\left(x ; \theta_{0}\right)}{\partial \theta} d T_{n}^{(i)}(x)+ \\
& +u \sqrt{n} \int_{-\infty}^{\infty} \frac{\partial \log t\left(x ; \theta_{0}\right)}{\partial \theta} d\left[T_{n}^{(-1)}(x)+T_{n}^{(0)}(x)\right]--\frac{u^{2}}{2} J\left(\theta_{0}\right)+o_{p}(1) .
\end{aligned}
$$

Proof of the Lemma 3.2. We expand the integrands in LRS $L_{n}(u)$ in Taylor series on powers of $\xi_{n i}(x)=\left[\frac{t^{(i)}\left(x ; \theta_{n}\right)}{t^{(i)}\left(x ; \theta_{0}\right)}\right]^{1 / 2}-1$ and $\eta_{n}(x)=\left[\frac{t\left(x ; \theta_{n}\right)}{t\left(x ; \theta_{0}\right)}\right]^{1 / 2}-1$. Then we obtain, respectively

$$
\begin{aligned}
& \log \left[\frac{t^{(i)}\left(x ; \theta_{n}\right)}{t^{(i)}\left(x ; \theta_{0}\right)}\right]=2 \log \left(1+\xi_{n i}(x)\right)=2 \xi_{n i}(x)-\xi_{n i}^{2}(x)+\gamma_{n i}(x)\left|\xi_{n i}(x)\right|^{3}, \\
& \log \left[\frac{t\left(x ; \theta_{n}\right)}{t\left(x ; \theta_{0}\right)}\right]=2 \log \left(1+\eta_{n}(x)\right)=2 \eta_{n}(x)-\eta_{n}^{2}(x)+\beta_{n}(x)\left|\eta_{n}(x)\right|^{3},
\end{aligned}
$$

where $\left|\gamma_{n i}(x)\right|<1, i=\overline{1, k}$ and $\left|\beta_{n}(x)\right|<1$. In other hand, functions $\xi_{n i}(x), \eta_{n}(x)$ expand in the neighborhood of $\theta=\theta_{0}$. We have

$$
\xi_{n i}(x)=\frac{u}{2 \sqrt{n}} \frac{\partial \log t^{(i)}\left(x ; \theta_{0}\right)}{\partial \theta}-\frac{u^{2}}{8 n}\left[\left(\frac{\partial \log t^{(i)}\left(x ; \theta_{0}\right)}{\partial \theta}\right)^{2}++\frac{1}{t^{(i)}\left(x ; \theta_{0}\right)} \frac{\partial^{2} t^{(i)}\left(x ; \theta_{0}\right)}{\partial \theta^{2}}\right]+o\left(\frac{1}{n}\right), n \rightarrow \infty
$$

$i=1, \ldots, k$ and 


$$
\eta_{n}(x)=\frac{u}{2 \sqrt{n}} \frac{\partial \log t\left(x ; \theta_{0}\right)}{\partial \theta}-\frac{u^{2}}{8 n}\left[\left(\frac{\partial \log t\left(x ; \theta_{0}\right)}{\partial \theta}\right)^{2}+\frac{1}{t\left(x ; \theta_{0}\right)} \frac{\partial^{2} t\left(x ; \theta_{0}\right)}{\partial \theta^{2}}\right]+o\left(\frac{1}{n}\right), n \rightarrow \infty .
$$

It is easy to verify that for all $i=\overline{1, k}$,

$$
\begin{aligned}
& \xi_{n i}^{2}(x)=\frac{u^{2}}{4 n}\left(\frac{\partial \log t^{(i)}\left(x ; \theta_{0}\right)}{\partial \theta}\right)^{2}+o\left(\frac{1}{n}\right), n \rightarrow \infty, \quad\left|\xi_{n i}(x)\right|^{3}=o\left(\frac{1}{n}\right), n \rightarrow \infty, \\
& \eta_{n}^{2}(x)=\frac{u^{2}}{4 n}\left(\frac{\partial \log t\left(x ; \theta_{0}\right)}{\partial \theta}\right)^{2}+o\left(\frac{1}{n}\right), n \rightarrow \infty, \quad\left|\eta_{n}(x)\right|^{3}=o\left(\frac{1}{n}\right), n \rightarrow \infty,
\end{aligned}
$$

Thus, when $n \rightarrow \infty$

$$
\begin{aligned}
& 2 n \sum_{i=1}^{k} \int_{-\infty}^{\infty} \xi_{n i}(x) d T_{n}^{(i)}(x)+2 n \int_{-\infty}^{\infty} \eta_{n}(x) d\left[T_{n}^{(-1)}(x)+T_{n}^{(0)}(x)\right]=u \sum_{i=1}^{k} \sqrt{n} \int_{-\infty}^{\infty} \frac{\partial \log t^{(i)}\left(x ; \theta_{0}\right)}{\partial \theta} d T_{n}^{(i)}(x)+ \\
& +u \sqrt{n} \int_{-\infty}^{\infty} \frac{\partial \log t\left(x ; \theta_{0}\right)}{\partial \theta} d\left[T_{n}^{(-1)}(x)+T_{n}^{(0)}(x)\right]-\frac{u^{2}}{4}\left|R_{1 n}-R_{2 n}\right|+o_{p}(1)
\end{aligned}
$$

where

$$
\begin{aligned}
& R_{1 n}=\sum_{i=1}^{k} \int_{-\infty}^{\infty}\left(\frac{\partial \log t^{(i)}\left(x ; \theta_{0}\right)}{\partial \theta}\right)^{2} d T_{n}^{(i)}(x)+\int_{-\infty}^{\infty}\left(\frac{\partial \log t\left(x ; \theta_{0}\right)}{\partial \theta}\right) d\left[T_{n}^{(-1)}(x)+T_{n}^{(0)}(x)\right], \\
& R_{2 n}=\sum_{i=1}^{k} \int_{-\infty}^{\infty} \frac{\partial^{2} t^{(i)}\left(x ; \theta_{0}\right)}{\partial \theta^{2}} \frac{d T_{n}^{(i)}(x)}{t^{(i)}\left(x ; \theta_{0}\right)}+\int_{-\infty}^{\infty} \frac{\partial^{2} t\left(x ; \theta_{0}\right)}{\partial \theta^{2}} \frac{d\left[T_{n}^{(-1)}(x)+T_{n}^{(0)}(x)\right]}{t\left(x ; \theta_{0}\right)} .
\end{aligned}
$$

Note that $E_{\theta_{0}} R_{1 n}=J\left(\theta_{0}\right)$ and in view of $T^{(-1)}(+\infty ; \theta)+T^{(0)}(+\infty ; \theta)+\sum_{i=1}^{k} T^{(i)}(+\infty ; \theta)=1$, $E_{\theta_{0}} R_{2 n}=0$. Consequently, according to the law of large numbers for $n \rightarrow \infty$

$$
\left|R_{1 n}-J\left(\theta_{0}\right)\right|=o_{p}(1),\left|R_{2 n}\right|=o_{p}(1)
$$

Similarly for $n \rightarrow \infty$ we have

$$
\left|n \int_{-\infty}^{\infty} \zeta_{n}^{2}(x) d T_{n}^{(-1)}(x)+n \int_{-\infty}^{\infty} \eta_{n}^{2}(x) d\left[T_{n}^{(0)}(x)+T_{n}^{(-1)}(x)\right]--\frac{u^{2}}{4} J\left(\theta_{0}\right)\right|=o_{p}(1),
$$

and

$$
\left.\left|n \int_{-\infty}^{\infty} \beta_{n}(x)\right| \eta_{n}(x)\right|^{3} d\left[T_{n}^{(-1)}(x)+T_{n}^{(0)}(X)\right]+n \sum_{i=1}^{k} \int_{-\infty}^{\infty} \gamma_{n i}(x)\left|\xi_{n i}(x)\right|^{3} d T_{n}^{(i)}(x) \mid=o_{p}(1) .
$$

Now (21)-(26) implies (20). Lemma 3.2 is proved. 
Proof of the Theorem 3.1 is uses Lemmas 3.1 and 3.2, is fully conducted through the proof of Theorem in [4], using also Theorem A and therefore details are omitted.

\section{Conclusion}

Asymptotic theory of estimation and hypothesis testing is entirely based on the asymptotic properties of the LRS. The most important property of LRS is LAN, which enables the development of the asymptotic theory of maximum likelihood and Bayesian type estimators and contiguity of probability measures. In this paper proved the LAN property of LRS in CRM under random censoring from both sides. These results can be used to find the limiting distributions of maximum likelihood and the Bayesian type estimates in considered model.

\section{References}

1. Abdushukurov A.A. The model of random censorship from both sides and the criteria of independence for her // Doclady Acad. Nauk. Uzbekistan. 1994. №11. p.8-9. (In Russian).

2. Abdushukurov A.A. Estimators of unknown distributions from incomplete observations and its properties. LAMBERT Academic Publishing. 2011. 299 p. (In Russian).

3. Abdushukurov A.A., Nurmuhamedova N.S. Approximation of the Likelihood Ratio Statistics in competing risks model. // Doclady Acad. Nauk. Uzbekistan. 2012. N.3. (In Russian, to appear).

4. Abdushukurov A.A., Nurmuhamedova N.S. Approximation of the likelihood ratio statistics in competing risks model under random censorship from both sides // ACTA NUUz. 2011. N.4. p.162-172. (In Russian).

5. Bobojonov J.A., Nurmuhamedova N.S. // Approximation of the likelihood ratio statistics in competing risks model under random censorship from the right // In Statistical Methods of Estimation and Hyphoteses Testing. Perm State University. Perm. 2011, Issue 23, p. 152-165. (In Russian)

6. Wald A. Tests of statistical hypothesis concerning several parameters, when the number of observations is large// Trans. Amer. Math. Soc. 54. 1943. p.426-482.

7. Le Cam L. On some asymptotic properties of the maximum likelihood estimates and related Bayes estimates.// Univ. California Publ. Statist. 1953. v.1. p. 277330.

8. Hajek J. Local asymptotic minimax and admissibility in estimation // Proc. Sixth. Berkeley Symp. on Math. Statist. and Prob. -1972. -V.1. -P. 175-194.

9. Rusas J. Contiguity of probability measures. -M.: Mir. 1975. 254p. (In Russian).

10. Ibragimov I.A, Khas'minskii R.Z. Asymptotic estimation theory. -M.: Nauka. 1979. 527p. (In Russian).

11. Van der Vaart A.W. Asymptotic Statistics. Cambridge Univ. Press. 1998. 443p. 